\title{
Assessing the Effect of Piperacillin/Tazobactam on Hematological Parameters in Patients Admitted with Moderate or Severe Foot Infections
}

\author{
Will Fry · Sean McCafferty · Catherine Gooday • Ian Nunney • \\ Ketan K. Dhatariya (D)
}

Received: October 31, 2017 / Published online: January 4, 2018

(c) The Author(s) 2018. This article is an open access publication

\begin{abstract}
Introduction: Piperacillin/tazobactam is a commonly used antibiotic for the empirical treatment of severe diabetic foot infections. One of the most feared complications of this drug is the development of pancytopenia. The aim of this study was to determine whether the use of piperacillin/tazobactam caused any hematological changes in patients admitted with severe diabetes-related foot infections from a specialist multidisciplinary foot clinic. Specifically, looking at whether it caused anemia, leukopenia, neutropenia, or thrombocytopenia. Methods: A 1-year retrospective analysis of patients admitted to a tertiary care center for treatment of diabetes-related foot infection
\end{abstract}

Enhanced content To view enhanced content for this article go to http://www.medengine.com/Redeem/ 790DF06047054999.

Electronic supplementary material The online version of this article (https://doi.org/10.1007/s13300017-0357-1) contains supplementary material, which is available to authorized users.

W. Fry · S. McCafferty · C. Gooday · I. Nunney ·

K. K. Dhatariya ( $\square)$

Norwich Medical School, University of East Anglia,

Norwich, Norfolk, UK

e-mail: ketan.dhatariya@nnuh.nhs.uk

C. Gooday · K. K. Dhatariya

Elsie Bertram Diabetes Centre, Norfolk and Norwich University Hospitals NHS Foundation Trust, Colney

Lane, Norwich, Norfolk NR4 7UY, UK using piperacillin/tazobactam. Hematological indices, urea and electrolytes, and C-reactive protein (CRP) were recorded pretreatment, during treatment, and posttreatment. HbA1c, vitamin $B_{12}$, folate, thyroid-stimulating hormone, and free thyroxin were also analyzed to exclude any potential confounders as a cause of pancytopenia.

Results: A total of 154 patients were admitted between 1 January 2016 and 31 December 2016 who received piperacillin/tazobactam for severe diabetes-related foot infection. On admission, white cell count and CRP were raised and fell significantly within the first $48 \mathrm{~h}$. Other hematological factors did not change. Five patients developed a mild pancytopenia, of which three were unexplained.

Conclusion: In this relatively small cohort, pancytopenia did not occur. As such, piperacillin/tazobactam appeared to have a low risk of adverse hematological outcomes and remains the treatment of choice for severe diabetes-related foot infections.

Keywords: Diabetic foot infection; Piperacillin/tazobactam; Pancytopenia

\section{INTRODUCTION}

In 2015, worldwide, the prevalence of diabetes in adults aged between 20 and 79 years old was estimated at 415 million people, at an annual 
global cost of approximately US $\$ 1.3$ trillion $[1,2]$. A significant part of this cost was due to acute hospital admissions. Diabetic foot infections are common, and are the most common reason for a "diabetes-specific" acute hospital admission, accounting for the most hospital days of all diabetes-related complications [3, 4].

Most moderate to severe diabetic foot infections are due to polymicrobial colonization of tissue by Gram-positive cocci, aerobic Gram negative bacilli, and anaerobes [5-7]. As such, the empirical use of broad-spectrum antibiotics prior to sensitivities being available have proved to be clinically effective, with reported rates of clinical resolution between $77 \%$ and $94 \%$ [5, 8-10]. Piperacillin/tazobactam $\left(\right.$ Tazocin $^{\circledR}$, Pfizer Ltd, Sandwich, UK) is an antibiotic containing the extended-spectrum agent piperacillin together with the $\beta$-lactamase inhibitor tazobactam. The drug has activity against a wide variety of Gram-positive and Gram-negative organisms and Pseudomonas aeruginosa. Because of its extended spectrum, the drug has been shown to be effective in treating moderate to severe diabetic foot infections [5-13], showing an advantage over ertapenem [5]. Consequently, piperacillin/tazobactam is commonly recommended as first-line empirical treatment owing to its high efficacy and relative safety compared with other antibiotics [8, 12, 14-16].

The manufacturer's package leaflet for piperacillin/tazobactam [15] has documented that pancytopenia is a side effect of the drug, with the British National Formulary (BNF) also stating this adverse drug reaction [17]. Evidence from both sources imply that pancytopenia is uncommon, with the manufacturer declaring that the frequency cannot be determined from the available data, and the BNF as "very rarely".

Case reports have also inferred the relationship between piperacillin/tazobactam and hematological changes, which is caused by reversible bone marrow suppression. Several case reports and cohort studies [18-25] as well as a systematic review [26] have shown patients to have neutropenia, leukopenia, agranulocytosis, or pancytopenia associated with treatment by piperacillin/tazobactam. However, evidence suggests that this is dose and duration dependent [25].
There is little evidence regarding hematological complications specifically in diabetic foot infection patients receiving piperacillin/tazobactam. The current literature evidence consists of case reports [19]. A systematic review of piperacillin-induced neutropenia found that whilst neutropenia was a rare complication in patients receiving piperacillin for over 15 days, the authors could not rule out hematological complications occurring prior to 15 days and that this required further investigation [26].

The significance of this adverse effect is considerable because pancytopenia in diabetic foot patients could worsen their infection and increase patient mortality [27]. The threat of pancytopenia could also act as a potential deterrent for the routine use of piperacillin/tazobactam.

The aim of this study is to assess whether hematological changes/pancytopenia occurs in patients with diabetic foot infections treated with piperacillin/tazobactam and to evaluate the appropriateness of this commonly used antibiotic.

\section{METHODS}

This study was a 1-year retrospective analysis of all patients admitted to the Norfolk and Norwich University Hospitals NHS Foundation Trust (Norwich, UK) between 1 January 2016 and 31 December 2016 for treatment of diabetic foot infections treated with piperacillin/tazobactam. This was a pragmatic study using a convenience sample.

After completion of the intravenously administered piperacillin/tazobactam course, orally administered co-amoxiclav $625 \mathrm{mg}$ three times daily was prescribed to the majority of patients, as per the local guidelines for diabetic foot infections [14]. Once antimicrobial sensitivities were known, the piperacillin/tazobactam was changed to a variety of other antibiotics. These included ciprofloxacin $500 \mathrm{mg}$ twice daily; metronidazole $400 \mathrm{mg}$ three times daily; trimethoprim $200 \mathrm{mg}$ twice daily; vancomycin $1 \mathrm{~g}$ as a one-off dose and then dosed according to plasma concentrations; 
doxycycline $200 \mathrm{mg}$ once daily; gentamicin dosed according to body weight and flucloxacillin $500 \mathrm{mg}$ four times daily.

All patients admitted with diabetic foot infections are seen by the inpatient multidisciplinary specialist diabetes foot team and their details kept electronically. Medication used was assessed using the E-Prescribing Medicines Administration (EPMA) system (JAC Medicines Management, Basildon, UK). Using the date of admission, we interrogated EPMA to identify patients receiving piperacillin/tazobactam for their diabetes-related foot infection; the date of administration and dose were noted. Patients were also excluded if they had no blood test results from the date of piperacillin/tazobactam administration to 28 days post administration.

Blood test results were recorded from the hospital's central pathology system Sunquest Integrated Clinical Environment (ICE) (Sunquest Information Systems, Uxbridge UK). Pretreatment values (including single values from any of the 3 days before administration), those taken during piperacillin/tazobactam administration (day 1-7), and posttreatment values [14 days after day 7 ( \pm 7 days)] were recorded. This length of follow-up was chosen because of previous data showing that the medial length of stay using our previous published antibiotic regimen was 9.25 days (IQR 2-25) [14]. In addition, glycated hemoglobin (HbA1c), vitamin $B_{12}$, folate, thyroid-stimulating hormone, and free thyroxin were also recorded pre and post administration, in order to exclude any potential confounders causing pancytopenia.

The hospital electronic letters template was also interrogated to check for potentially confounding causes of hematological abnormalities.

This was a retrospective case notes analysis study and as such the Norfolk and Norwich University Hospitals NHS Foundations Trust audit department designated this as a service improvement exercise and ethical approval was deemed unnecessary. This article is based on previously conducted studies and does not involve any new studies of human or animal subjects performed by any of the authors.

\section{RESULTS}

The Consort diagram showing patient selection is shown in Fig. 1. Of the 154 people included in the analysis, only 38 were female. The cohort had a mean age of 69 years $(\mathrm{SD} \pm 14.46)$. Of these 154 patients receiving piperacillin/tazobactam, 131 patients received $4.5 \mathrm{~g}$ three times daily while 22 patients received $4.5 \mathrm{~g}$ twice daily, all via intravenous infusion. One patient received a single dose only at the time of admission. The mean duration of piperacillin/tazobactam administration was 5.3 days $(\mathrm{SD} \pm 3.3)$.

Data were collated giving the mean values at each time point, standard deviations, and the number of data points were calculated. These

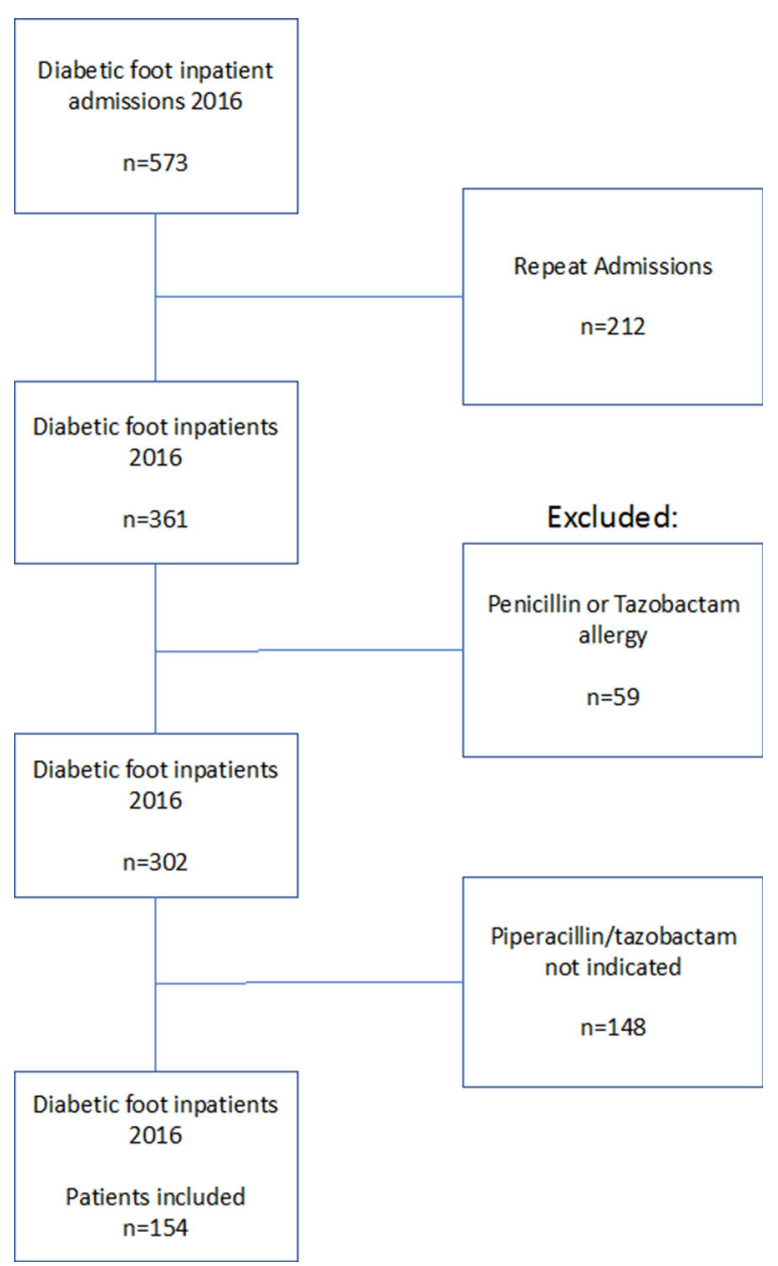

Fig. 1 Consort diagram illustrating the study of patients into the study 
data are presented in Table 1 and graphically in the supplementary materials.

A non-specific marker of infection, CRP, was used to indicate the progress of the patient's diabetic foot infection during piperacillin/tazobactam treatment. Figure 2 shows an initial rise in CRP 2 days after initiation of treatment. This is followed by a fall, as well as improvements in all of the other measured hematological values over the remaining duration of piperacillin/tazobactam administration and follow-up (Table 1 or Supplementary Figs. A-E).

\section{Hematological Parameters}

The mean lymphocyte count dropped initially, reaching a nadir after $48 \mathrm{~h}$. It then rose reaching a peak at 7 days. Basophil concentrations remained low for the first 4 days after admission before rising to a maximum by 7 days. Monocyte concentrations remained broadly unchanged from the time of admission for $48 \mathrm{~h}$, and then fell for a further $48 \mathrm{~h}$ before returning to values similar to those on admission by 7 days. Eosinophil concentrations dropped slightly in the first $24 \mathrm{~h}$ following admission, but then steadily rose to reach a maximum by 7 days. The mean red blood cell count rose after the first $24 \mathrm{~h}$ admission and then fell to a nadir at 4 days after admission before returning to values similar to those on admission by 7 days. Mean corpuscular hemoglobin rose to reach a maximum by $24 \mathrm{~h}$ and then fell to reach a nadir by 7 days. Values at 14 days were similar to those at 7 days. Mean corpuscular volume was generally unchanged during the whole admission.

Hematological parameters were evaluated for mean values outside of the reference range. For the purposes of this study, mean values below the lower limit of normal were evaluated to assess for pancytopenia or individual abnormalities of that particular parameter. Results above the upper limit of normal were to be expected in a normal response to a bacterial infection. Table 1 and Supplementary Figs. A-E show that all values improved from the peak of infection on day 2 to 14 days posttreatment, with all of them being within the reference ranges by 14 days. Most of these parameters also remained within the reference range throughout the study period, with exceptions of those that might be expected with an infectionwhite blood cells (WBC), lymphocytes, and monocytes.

The data showed that on admission 136 patients had a hemoglobin concentration below the lower limit of the reference range (i.e., $130 \mathrm{~g} / \mathrm{L}), 79$ of whom had a hemoglobin concentration of below $100 \mathrm{~g} / \mathrm{L}$. Twenty-two patients had a platelet count below the lower limit of the reference range (i.e., $150 \times 10^{9} / \mathrm{L}$ ). The data also showed that six patients had one or more component of the WBC below the lower limit of the reference range. Five patients were highlighted to have low hemoglobin, white cells, and platelets, suggesting pancytopenia at some stage during their admission. Of these, two patients had potential causes for this. A 65-year-old man had known follicular lymphoma and had had a stem cell transplant prior to admission for the diabetic foot infection. His baseline values prior to the administration of piperacillin/tazobactam were all low, as were subsequent values at 14-day follow up. The other patient was a 73-year-old man taking darbepoetin alfa $\left(\right.$ Aranesp $^{\circledR}$ ) for chronic renal failure. His WBC and hemoglobin were persistently low, although the platelet count was only down on day 1, 2 and 14 days post termination of treatment.

The remaining three patients had no clear cause for their low values. A 68-year-old man receiving piperacillin/tazobactam for 7 days had a low hemoglobin concentration throughout the study period (between $119 \mathrm{~g} / \mathrm{L}$ day 5 and $89 \mathrm{~g} / \mathrm{L}$ at 14 days post treatment). However, both the WBC and the platelets remained within the reference range for the duration of administration only to fall below in the 14-day post treatment reading. A 63-year-old man had persistently low hemoglobin level (between $124 \mathrm{~g} / \mathrm{L}$ and $102 \mathrm{~g} / \mathrm{L}$ ) for the duration of his admission and low WBC on days 1, 2, and 6 $\left(3.8,3.0\right.$, and $3.1 \times 10^{9}$, respectively) and low platelets on days $2-6$ (between $123 \times 10^{9} / \mathrm{L}$ and $143 \times 10^{9} / \mathrm{L}$ ). An 84-year-old man receiving the antibiotic for 5 days had a similar pattern of persistently low hemoglobin with individual 


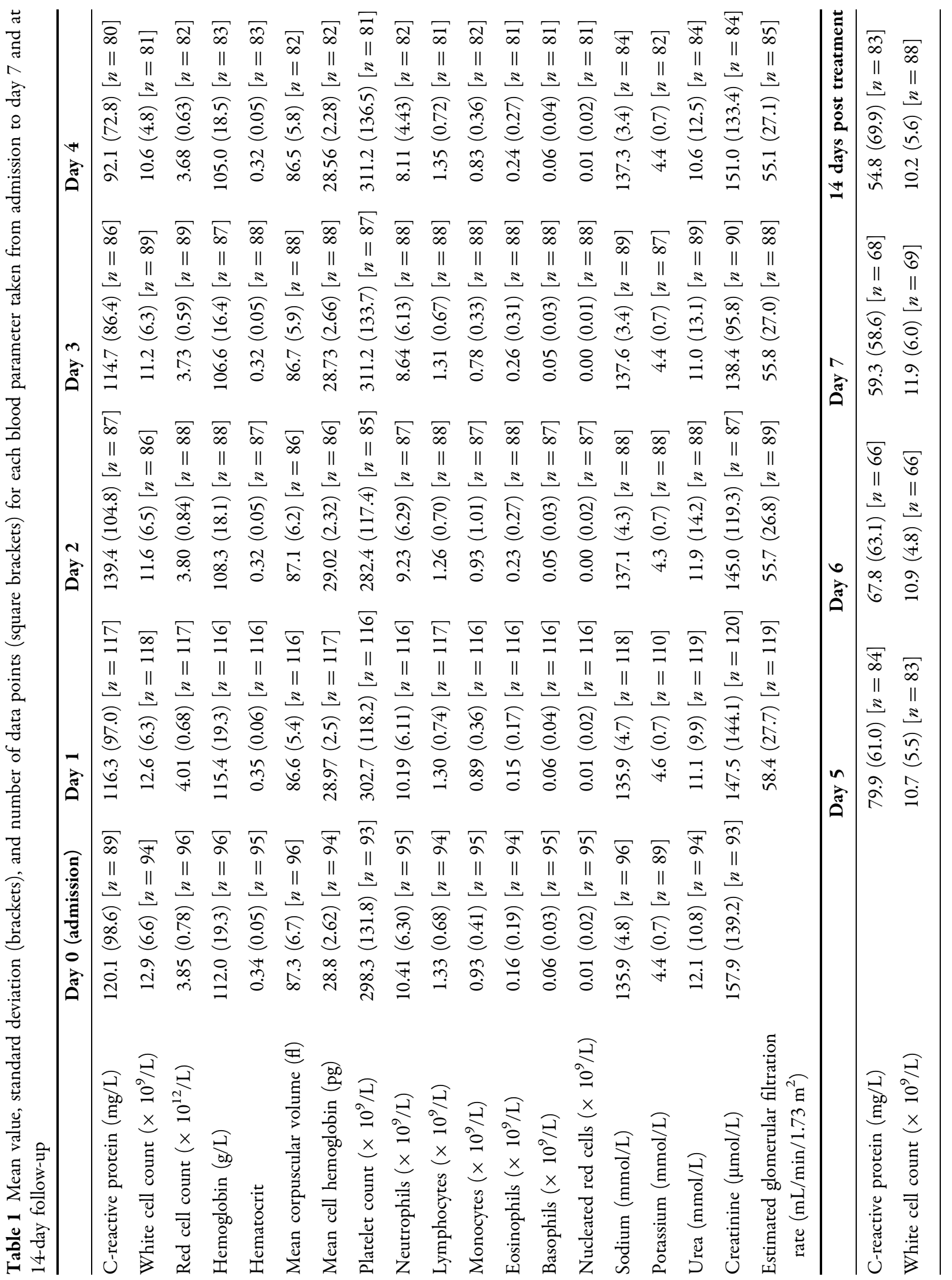




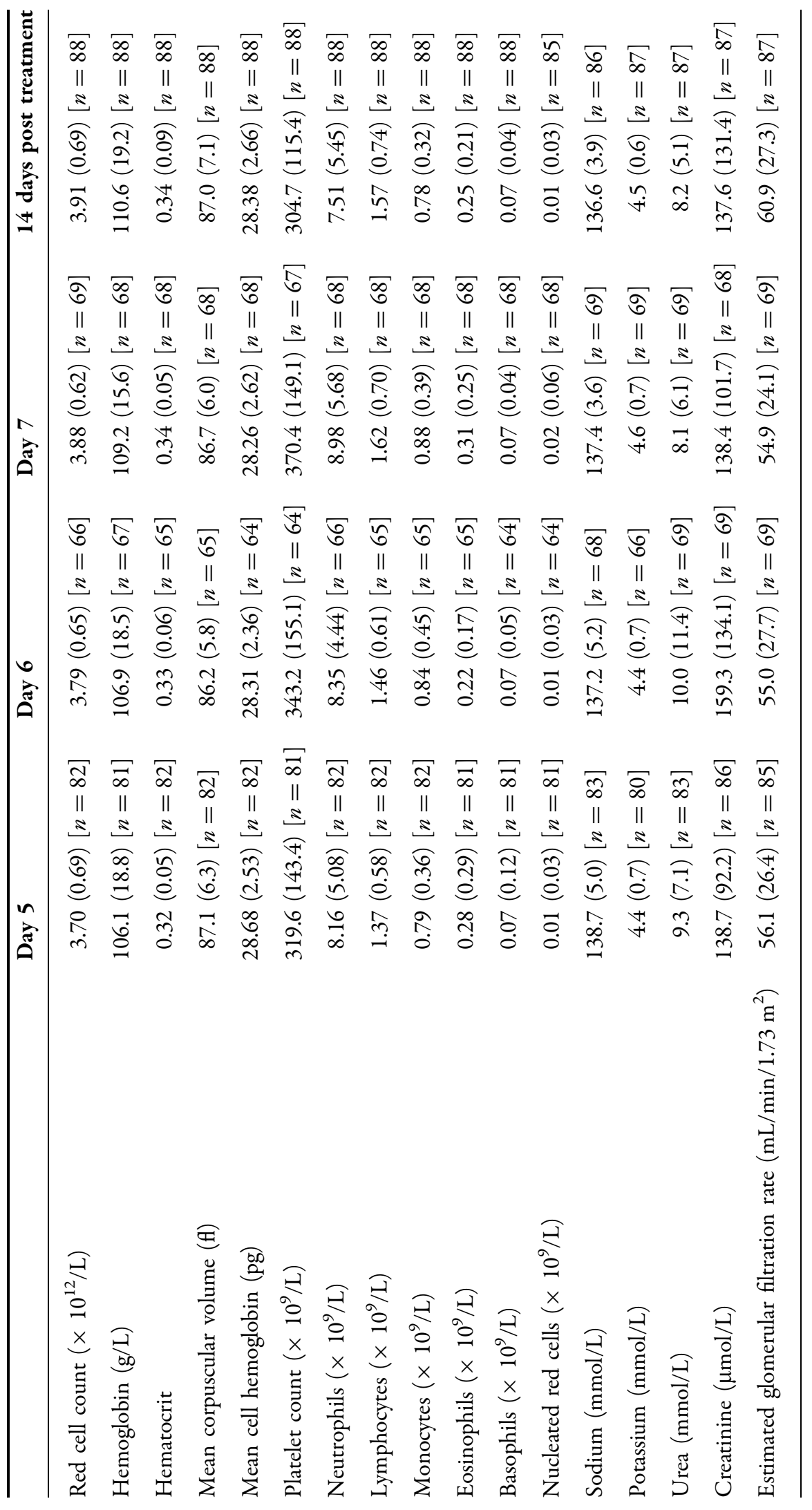




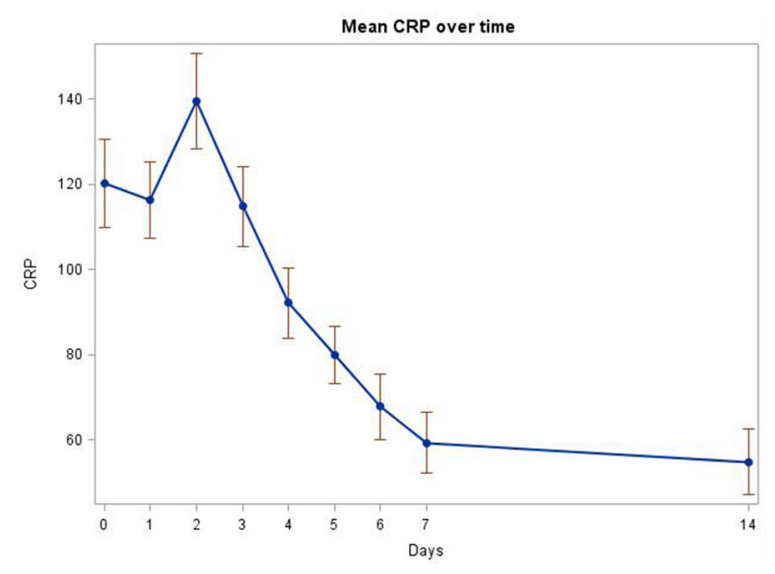

Fig. 2 Mean concentrations of C-reactive protein over time

low values for WBC $\left(3.6 \times 10^{9} / \mathrm{L}\right)$ and platelets $\left(145 \times 10^{9} / \mathrm{L}\right)$ on day 3 only.

\section{Renal Function}

Mean eGFR fell during the first $48 \mathrm{~h}$ following admission, and then remained broadly unchanged until day 7. By day 14 , however, eGFR had risen to above admission values. Serum sodium concentrations rose after the first $24 \mathrm{~h}$ following admission to reach a peak by 5 days before falling again by day 14 . In contrast, mean potassium concentrations rose during the first $24 \mathrm{~h}$ following admission before falling by day 2 and then rising by day 7 . Mean urea and creatinine concentrations fell from the time of admission to 7 days.

\section{DISCUSSION}

This study has shown that in a cohort of all admissions for a severe diabetic foot infection to a large tertiary specialist care center during a single calendar year, the use of piperacillin/tazobactam had no adverse effect on any measured hematological or inflammatory markers. In addition, the use of piperacillin/tazobactam led to rapid resolution of abnormal blood results which were associated with the underlying infection triggering the initial admission. This confirms the antibiotic's excellent broadspectrum cover, suggesting it is an effective and safe medication for the treatment of diabetesrelated foot infections. Furthermore, these findings justify the inclusion of piperacillin/tazobactam in specialist diabetic foot formularies [14].

These data have many strengths. One year of data was analyzed from a large regional center for treatment of severe diabetic foot infections. Patients were successfully followed up for 14 days, which directly addressed the concern that hematological abnormalities resulting from piperacillin/tazobactam administration were time dependent [25] and possible within the first 15 days [26]. The patients received piperacillin/tazobactam for an average of 5.3 days, and no patient exceeded 15 days of continuous treatment. As well as this, the same authors [25] suggested that the blood dyscrasias were dose dependent. Over $85 \%$ of the cohort in the current study received $4.5 \mathrm{~g}$ of piperacillin/tazobactam three times per day; 22 (13\%) patients received the antibiotic twice a day and one patient only having a stat dose. That this study was carried out in a single center, with all of the blood results being available also increases the validity of the findings, although we acknowledge that being a singlecenter study can also be seen as a limitation. The format of the study also provides strength. However, having rapid access to microbiological advice from the same consultant ensured consistency of care.

However, there are also several limitations. It is reported that hematological abnormalities, including pancytopenia, occur very rarely with the administration of piperacillin/tazobactam. The incidence has not been determined, with the manufacturer stating the frequency of this adverse effect is unknown [15] and the UK British National Formulary suggesting it occurs very rarely [17]. Evidence suggesting the link is largely made up from case studies which also imply that this adverse effect is uncommon [18-25]. Therefore, with a sample size of 154 patients it may be very difficult to detect this rare event. A larger potentially multicenter cohort is required to more accurately determine a correlation between piperacillin/tazobactam administration and the development of any hematological abnormalities. 
The present study followed up patients for 14 days post administration of piperacillin/tazobactam. No longer-term follow-up was performed. Evidence suggests that the correlation is dose and duration dependent [25]. However, it is possible that hematological abnormalities associated with the antibiotic occurred after the follow-up period, although this has not been reported. It would also be difficult to assess as blood results are not routinely taken after this time. However, because the mean length of administration was less than 6 days, and blood was taken up to 8 days later, with almost all normalizing by then, it was felt that the development of piperacillin/tazobactam-induced hematological abnormality beyond that time would be unlikely.

The results were analyzed as overall means of each parameter. However, where there were outliers the individual patient records were examined to look for any trends in other hematological abnormalities. None were identified.

There were several confounding factors that need to be taken into account, despite no abnormal blood results being found. These included patients being on other antibiotics depending on antimicrobial sensitivities. These included ciprofloxacin, metronidazole, trimethoprim, vancomycin, gentamicin, flucloxacillin, and doxycycline. These medications have the capacity to interfere with hematological values [17]. In addition, the local foot formulary suggests co-amoxiclav $625 \mathrm{mg}$ three times daily after piperacillin/tazobactam treatment [14]. This might have improved the follow-up results. Furthermore, whilst there were three patients who had a mild degree of pancytopenia during their treatment, we were unable to account for why they developed it. Whilst other potential causes of pancytopenia were sought in all patients-i.e., other medical conditions or the use of any drugs-by looking at discharge summaries, these are known to be inaccurate [28]. It may well be that these abnormalities were as a result of the piperacillin/tazobactam treatment. Several other patients were found to have conditions or be on medications that may have predisposed to developing pancytopenia, but all had normal hematological parameters. These included 16 patients with various conditions known to cause pancytopenia (hematological malignancy, medication (methotrexate, mycophenolate mofetil, and tacrolimus), hypothyroidism, liver disease, and renal failure).

In summary, NICE guidance recommends broad-spectrum antibiotics in the management of diabetes-related foot infections [29]. Whilst piperacillin/tazobactam has been very rarely associated with the development of hematological abnormalities, in particular pancytopenia, the present study failed to show this. Thus piperacillin/tazobactam remains an effective and safe medication for these patients. However, much larger studies are needed to determine the incidence of this very rare complication.

\section{ACKNOWLEDGEMENTS}

Funding. No funding or sponsorship was received for this study or publication of this article.

Authorship. Will Fry and Sean McCafferty collected the data and wrote the first draft of the manuscript. Ketan K. Dhatariya and Catherine Gooday had the initial idea and wrote the final draft of the manuscript. Ian Nunney did the data analysis. All authors saw and approved the final submitted manuscript. All named authors meet the International Committee of Medical Journal Editors (ICMJE) criteria for authorship for this manuscript, take responsibility for the integrity of the work as a whole, and have given final approval for the version to be published.

Disclosures. Will Fry, Sean McCafferty, Catherine Gooday, Ian Nunney, and Ketan K. Dhatariya have nothing to disclose.

Compliance with Ethics Guidelines. This article is based on previously conducted studies and does not involve any new studies of human or animal subjects performed by any of the authors. 
Open Access. This article is distributed under the terms of the Creative Commons Attribution-NonCommercial 4.0 International License (http://creativecommons.org/licenses/ by-nc/4.0/), which permits any noncommercial use, distribution, and reproduction in any medium, provided you give appropriate credit to the original author(s) and the source, provide a link to the Creative Commons license, and indicate if changes were made.

\section{REFERENCES}

1. Ogurtsova K, da Rocha Fernandes JD, Huang Y, et al. IDF Diabetes Atlas: global estimates for the prevalence of diabetes for 2015 and 2040. Diabetes Res Clin Pract. 2017;128:40-50.

2. Bommer C, Heesemann E, Sagalova V, et al. The global economic burden of diabetes in adults aged 20-79 years: a cost-of-illness study. Lancet Diabetes Endocrinol. 2017;5(6):423-30.

3. NHS Digital. National Diabetes Inpatient Audit (NaDIA). 2016. http://www.content.digital.nhs.uk/ catalogue/PUB23539. 2017. Accessed 20 Nov 2017.

4. Lipsky BA, Berendt AR, Embil J, de Lalla F. Diagnosing and treating diabetic foot infections. Diabetes Metab Res Rev. 2004;20(S1):S56-64.

5. Xu ZR, Ran XW, Xian Y, et al. Ertapenem versus piperacillin/tazobactam for diabetic foot infections in China: a phase 3, multicentre, randomized, double-blind, active-controlled, non-inferiority trial. J Antimicrob Chemother. 2016;71(6):1688-96.

6. Crouzet J, Lavigne JP, Richard JL, Sotto A. Diabetic foot infection: a critical review of recent randomized clinical trials on antibiotic therapy. Int J Infect Dis. 2011;15(9):e601-10.

7. Lipsky BA, Berendt AR, Cornia PB, et al. 2012 Infectious Diseases Society of America clinical practice guideline for the diagnosis and treatment of diabetic foot infections. Clin Infect Dis. 2012;54(12):e132-73.

8. Lipsky BA, Armstrong DG, Citron DM, et al. Ertapenem versus piperacillin/tazobactam for diabetic foot infections (SIDESTEP): prospective, randomised, controlled, double-blinded, multicentre trial. Lancet. 2005;366(9498):1695-703.

9. Lipsky BA, Holroyd KJ, Zasloff M. Topical versus systemic antimicrobial therapy for treating mildly infected diabetic foot ulcers: a randomized, controlled, double-blinded, multicenter trial of pexiganan cream. Clin Infect Dis. 2008;47(12):1537-45.

10. Lipsky BA, Giordano P, Choudhri S, Song J. Treating diabetic foot infections with sequential intravenous to oral moxifloxacin compared with piperacillin/tazobactam/amoxicillin-clavulanate. J Antimicrob Chemother. 2007;60(2):370-6.

11. Tan JS, Wishnow RM, Talan DA, Duncanson FP, Norden CW. Treatment of hospitalized patients with complicated skin and skin structure infections: double-blind, randomized, multicenter study of piperacillin-tazobactam versus ticarcillin-clavulanate. The Piperacillin/Tazobactam Skin and Skin Structure Study Group. Antimicrob Agents Chemother. 1993;37(8):1580-6.

12. Harkless L, Boghossian J, Pollak R, et al. An openlabel, randomized study comparing efficacy and safety of intravenous piperacillin/tazobactam and ampicillin/sulbactam for infected diabetic foot ulcers. Surg Infect. 2005;6(1):27-40.

13. Saltoglu N, Dalkiran A, Tetiker $\mathrm{T}$, et al. Piperacillin/tazobactam versus imipenem/cilastatin for severe diabetic foot infections: a prospective, randomized clinical trial in a university hospital. Clin Microbiol Infect. 2010;16(8):1252-7.

14. Gooday C, Hallam C, Sieber C, et al. An antibiotic formulary for a tertiary care foot clinic: admission avoidance using intramuscular antibiotics for borderline foot infections in people with diabetes. Diabetic Med. 2013;30(5):581-9.

15. Pfizer Ltd. Tazocin $2 \mathrm{~g} / 0.25 \mathrm{~g}$ powder for solution for infusion. http://www.medicines.org.uk/emc/ medicine/2239. 2017. Accessed 20 Nov 2017.

16. Lipsky BA, Silverman MH, Joseph WS. A proposed new classification of skin and soft tissue infections modeled on the subset of diabetic foot infection. Open Forum Infect Dis. 2017;4(1):ofw255.

17. British Medical Association. Royal Pharmaceutical Society of Great Britain. London: British National Formulary; 2017.

18. Nguyen VD, Tourigny JF, Roy R, Brouillette D. Rapid-onset thrombocytopenia following piperacillin-tazobactam reexposure. Pharmacother. 2015;35(12):e326-30.

19. Uzen G, Onem Y, Hatipoglu M, et al. Piperacillin/tazobactam-induced neutropenia, thrombocytopenia, and fever during treatment of a diabetic foot infection. Scand J Infect Dis. 2013;45(1):73-6.

20. Reichardt P, Handrick W, Linke A, Schille R, Kiess W. Leukocytopenia, thrombocytopenia and fever 
related to piperacillin/tazobactam treatment: a retrospective analysis in 38 children with cytic fibrosis. Infection. 1999;27(6):355-6.

21. Perez-Vazquez A, Pastor JM, Riancho JA. Immune thrombocytopenia caused by piperacillin/tazobactam. Clin Infect Dis. 1998;27(3):650-1.

22. Gerber L, Wing EJ. Life-threatening neutropenia secondary to piperacillin/tazobactam therapy. Clin Infect Dis. 1995;21(4):1047-8.

23. Ruiz-Irastorza GU, Barreiro GU, Aguirre CI. Reversible bone marrow depression by high-dose piperacillin/tazobactam. $\mathrm{Br} \mathrm{J}$ Haematol. 1996;95(4):611-2.

24. Behbahani R, Kostman JR. Hypersensitivity reaction during prolonged use of piperacillin/tazobactam in treatment of osteomyelitis. Ann Pharmacother. 1995;29(9):936-7.

25. Peralta FG, Sanchez M, Roiz MP, et al. Incidence of neutropenia during treatment of bone-related infections with piperacillin-tazobactam. Clin Infect Dis. 2003;37(11):1568-72.

26. Scheetz MH, McKoy JM, Parada JP, et al. Systematic review of piperacillin-induced neutropenia. Drug Saf. 2007;30(4):295-306.

27. Seboxa T, Amogne W, Abebe W, et al. High mortality from blood stream infection in Addis Ababa, Ethiopia, is due to antimicrobial resistance. PLoS One. $2015 ; 10(2): \mathrm{e} 0144944$.

28. Daultrey H, Gooday C, Dhatariya K. Increased length of inpatient stay and poor clinical coding: audit of patients with diabetes. JRSM Short Rep. 2011;2(11):83.

29. National Institute for Health and Care Excellence. Diabetic foot problems: prevention and management. NG19. http://www.nice.org.uk/guidance/ ng19. 2015. Accessed 20 Nov 2017. 